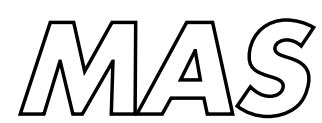

Modelling, Analysis and Simulation

\footnotetext{
Modelling, Analysis and Simulation 
Centrum voor Wiskunde en Informatica (CWI) is the national research institute for Mathematics and Computer Science. It is sponsored by the Netherlands Organisation for Scientific Research (NWO).

CWI is a founding member of ERCIM, the European Research Consortium for Informatics and Mathematics.

CWI's research has a theme-oriented structure and is grouped into four clusters. Listed below are the names of the clusters and in parentheses their acronyms.

Probability, Networks and Algorithms (PNA)

Software Engineering (SEN)

\section{Modelling, Analysis and Simulation (MAS)}

Information Systems (INS)

Copyright (C) 2006, Stichting Centrum voor Wiskunde en Informatica

P.O. Box 94079, 1090 GB Amsterdam (NL)

Kruislaan 413, 1098 SJ Amsterdam (NL)

Telephone +31205929333

Telefax +31205924199

ISSN 1386-3703 


\title{
Nonlinear instability and saturation of linearly stable current-carrying pair plasmas
}

\begin{abstract}
The nonlinear instability of current-carrying pair plasmas is investigated with a Vlasov-Poisson model for the two particle species. It is shown that linearly stable configurations are unstable against small incoherent perturbations of the particle distribution functions. The instability gives rise to a self-acceleration and growth of phase space holes. After the growth of the phase-space holes, the instability reaches a chaotic saturation where the finite-amplitude holes interact and merge, and after a long time, the system attains a stable equilibrium state with a smaller drift and a larger temperature than the initial one, and where a few stable phase-space holes are present.
\end{abstract}

2000 Mathematics Subject Classification: 76X05

Keywords and Phrases: pair plasma; nonlinear instability; BGK modes 



\title{
Nonlinear instability and saturation of linearly stable current-carrying pair plasmas
}

\author{
A. Luque ${ }^{1}$ H. Schamel ${ }^{2}$ B. Eliasson, ${ }^{1}$ and P.K. Shukla ${ }^{1}$ \\ ${ }^{1}$ Institut für Theoretische Physik IV, \\ Fakultät für Physik und Astronomie, \\ Ruhr-Universität Bochum, D-44780 Bochum, Germany \\ 2 Physikalisches Institut, Universität Bayreuth, D-95440 Bayreuth, Germany
}

(Dated: February 22, 2006)

The nonlinear instability of current-carrying pair plasmas is investigated with a VlasovPoisson model for the two particle species. It is shown that linearly stable configurations are unstable against small incoherent perturbations of the particle distribution functions. The instability gives rise to a self-acceleration and growth of phase space holes. After the growth of the phase-space holes, the instability reaches a chaotic saturation where the finiteamplitude holes interact and merge, and after a long time, the system attains a stable equilibrium state with a smaller drift and a larger temperature than the initial one, and where a few stable phase-space holes are present.

PACS numbers: 52.35.Fp, 52.35.Sb, 52.27.Ep, 81.05.Tp

\section{INTRODUCTION}

Pair plasmas, consisting of two species with opposite charges and equal masses, are the subject of increasing interest among physicists [1]. The presence of such physical systems in environments like the early universe, neutron stars, active galactic nuclei and pulsar magnetospheres makes the understanding of pair plasma dynamics a relevant study for astrophysics. Although electronpositron pair plasmas have since long been available in laboratories [2-4], pair annihilation and radiation cooling by cyclotron emission do not allow life-times sufficiently long for the excitation and development of coherent structures. This drawback is not present in the recently available long-lived pair plasmas composed by single-charged fullerene molecules [5], which promise to play an important role in the understanding of the underlying principles of plasma dynamics and the testing of analytical and numerical models. An analytical treatment of equilibrium solutions in pair plasmas [6] has resulted in the finding and description of trapped particle equilibria characterized by a shape function and a nonlinear dispersion relation.

A further motivation for the study of pair plasma dynamics lies in the insight they provide into 
the dynamics of more general plasmas, often composed by species with very different masses $[7,8]$. One of the main difficulties in the numerical research of ordinary electron-ion plasmas is the large difference between the two involved timescales. An integration of the full kinetic equations of both species is therefore computationally very expensive and often masked by numerical limitations and simplifications (e.g. modelling one of the species by fluid dynamics $[9,10]$ ) have instead been used to investigate the long-term behavior. However, as we will show in this paper, these simplifications exclude interesting and decisive phenomena that appear only in the fully nonlinear kinetic description.

It were first observed in Particle-in-Cell (PIC) simulations in a series of landmarking papers by Dupree and coworkers [13], that a current-carrying Maxwellian plasma composed of two species with not too different masses is unstable far below the threshold predicted by the linear theory [11]. In this model, an external electric field $E_{D}$ is initially imposed which produces a drift between both species, but which is then removed to allow the pair-plasma to relax in the course of time. However, due to the limitations in computational resources at the time, the number of simulated particles was not large enough to rule out the possibility that the instability was an effect only of the coarseness of the numerical description. Furthermore, the model that they proposed turned out not to be able to cover the full kinetic dynamics of the involved mechanism. For these reasons, we believe that a reinvestigation of this stability problem is timely, especially due to its impact on the anomalous transport [10] in plasmas. On the other hand, the saturation of nonlinear electrostatic instabilities has not received much attention so far, in contrast with the linearly unstable regime [12].

In this paper, we show the results of Vlasov-Poisson (VP) simulations that indicate that an instability associated with the formation and acceleration of trapping structures is indeed an inherent property of collisionless current-carrying (pair) plasmas. We sketch a description of the generic mechanism involved in the destabilization of the plasma as a self-acceleration of trapping structures that differs from Dupree's model [14], and is able to explain the driving of some particles far outside their thermal range, as observed in our simulation. The saturation of the nonlinear instability is also investigated. We observe that after a turbulent regime, a final inhomogeneous stable steady state is reached in which equilibrium trapping structures are present. Particle trapping is hence not only associated with the saturation of a linear instability -the common picturebut it also turns out to be the underlying mechanism of a not yet completely understood kind of nonlinear instability.

The paper is organized as follows: in Section II we describe the equations that govern the 
dynamics of the system, as well as the numerical approach used to solve them. In Section III we present and comment the numerical results. Finally, Section IV contains a summary of our investigation and some conclusions we can extract from it.

\section{GOVERNING EQUATIONS AND NUMERICAL TREATMENT}

We consider a drifting collisionless pair plasma with equal temperatures for both species. The dynamics is governed by the $1 \mathrm{D}$ Vlasov-Poisson system

$$
\begin{gathered}
{\left[\partial_{t}+v \partial_{x} \pm \Phi^{\prime}(x, t) \partial_{v}\right] f_{\mp}(x, v, t)=0,} \\
\Phi^{\prime \prime}(x, t)=\int d v f_{-}-\int d v f_{+} \equiv n_{-}-n_{+},
\end{gathered}
$$

where space $x$, time $t$, velocity $v$, the distribution functions $f_{ \pm}$and electric potential $(\Phi)$ are in units of the Debye length $\lambda_{D}$, the inverse plasma frequency $\omega_{p}^{-1}$, the thermal speed $V_{T}, n_{0} / V_{T}$ and $T / e$, respectively. Here, $\lambda_{D}=\left(n_{0} e^{2} / \epsilon_{0} T\right)^{1 / 2}, \omega_{p}=\left(n_{0} e^{2} / \epsilon_{0} m\right)$ and $V_{T}=(T / m)^{1 / 2}$, where $n_{0}$ is the unperturbed particle density of each species, $e$ is the magnitude of the electron charge, $m$ is the mass and $T$ is the thermal energy of the two species in the unperturbed state. The distribution functions of the homogeneous, unperturbed state are in the center-of-mass frame $f_{0 \pm}=(1 / \sqrt{2 \pi}) \exp \left\{-\left(v \pm v_{D} / 2\right)^{2} / 2\right\}$, with $v_{D}$ being the drift speed between both species. A linearization of Eq. (1), considering small perturbations $f_{1}$ and small $\partial_{v} f_{1}$ on these distribution functions and a subsequent stability analysis [11], predicts that the system is stable for drift speeds smaller than $v_{D}^{\star} \approx 2.6$.

We integrate numerically Eqs. (1a) and (1b) by means of a Fourier-Hermite [15] code in which the distribution functions are expanded as

$$
f_{ \pm}(x, v, t)=\frac{1}{\sqrt{2 \pi}} e^{-\frac{1}{2}\left(v \pm v_{D} / 2\right)^{2}} \sum_{m=0}^{M} \frac{\operatorname{He}_{m}\left(v \pm v_{D} / 2\right)}{(m-1) ! !} \sum_{n=-N}^{N} k_{n m}^{ \pm}(t) e^{\frac{i n \pi x}{L}}
$$

where $\operatorname{He}_{m}(z)$ is the Hermite polynomial of order $m$ and $L$ is the length of the system, on which periodic boundary conditions are imposed. Inserting the decomposition (2) into Eq. (1) yields an evolution equation for each coefficient, which we solve with a fourth-order Runge-Kutta algorithm.

The Fourier-Hermite expansion allows us to directly integrate the VP system, thus avoiding the discretization errors associated with other common plasma simulation techniques, such as PIC modelling. Besides, the Hermite expansion in velocities is well suited for distribution functions not far away from the Maxwell-Boltzmann distribution, which would be exactly represented by $M=0$. 
We here use $M=800$ Hermite polynomials and $N=64$ Fourier modes. As closure conditions at $m=M$, we impose outflow conditions [15] that minimize recurrence effects due to filamentation in velocity space, thus allowing to achieve long time simulations. The effect of neglecting higher order Hermite modes is a certain coarse-graining of the velocity dependence of $f_{ \pm}$, acting as a small dissipation term. The exact form of this dissipation does not, however, rely on any physical model, which represents a drawback of Fourier-Hermite simulations.

There are many different ways of perturbing the homogeneous distribution functions. A common approach is to set as initial condition $f=f_{0}(1+\epsilon \sin k x)$. Then, as long as $\epsilon \ll 1$, the linear theory can be applied and all modes experience initially Landau damping when $v_{D}<v_{D}^{\star}$. Later, nonlinear effects come into play and a trapped particle equilibrium emerges, which survives timeasymptotically in the so-called nonlinear Landau damping scenario [16, 17]. The reason why initially Landau damping prevails lies, of course, in the fact that the initial perturbation satisfies the condition of applicability of linear theory $\left|\partial_{v} f_{1}\right| \ll\left|\partial_{v} f_{0}\right|$ because of the same $v$-dependence of $f_{0}$ and $f_{1}$. In course of time, the field energy is monotonically decreasing leaving a tiny BGK-like hole structure. We use instead incoherent perturbations that topologically change the distribution functions and, though small, do not satisfy the linear criterion. To achieve that, we simply add a random normal noise to each coefficient $k_{m n}$ in the expansion (2). Quasineutrality is ensured by maintaining $k_{00}^{ \pm}=1$. Note that this procedure may lead to the regions with $f_{ \pm}<0$. Those regions are however small if the perturbation is small, and in the simulations they disappear after a short time due to the numerical dissipation.

The expansion (2) allows also to restrict the space of admissible perturbations to those that do not alter the total energy of the system. To impose this restriction, we note that the only coefficients that contribute to the energy expression are $k_{01}^{ \pm}, k_{02}^{ \pm}$and $k_{n 0}^{ \pm}$, and therefore we do not add any noise to these coefficients. The total energy will therefore be the same in the perturbed and unperturbed states. In the simulations presented here, we restricted ourselves to such energypreserving (i.e. adiabatic) perturbations. Thus we are confident that the observed effects are not a result of an uncontrolled addition of energy. We checked however that when this restriction is removed, the evolution of the system is qualitatively similar but slightly faster.

\section{NUMERICAL RESULTS}

We run the simulation in a linearly stable regime with $v_{D}=2<v_{D}^{\star}$. Although the perturbation is small $\left(f_{1} / f_{0} \approx 0.08\right)$, the observed result is in clear contradiction with the linear theory and also 
with the nonlinear Landau damping scenario. The evolution of the system is displayed in Fig. 1, where the field energy is plotted as a function of time. At least three phases can be distinguished in the time evolution: (a) a transient damping phase resembling Landau damping but where some nonlinear phase-locking takes place which produces tiny coherent seed holes out of the noise (b) a growing nonlinear instability and (c) a saturation of the instability and a decay towards a new stable equilibrium. Let us study in detail each of these phases.

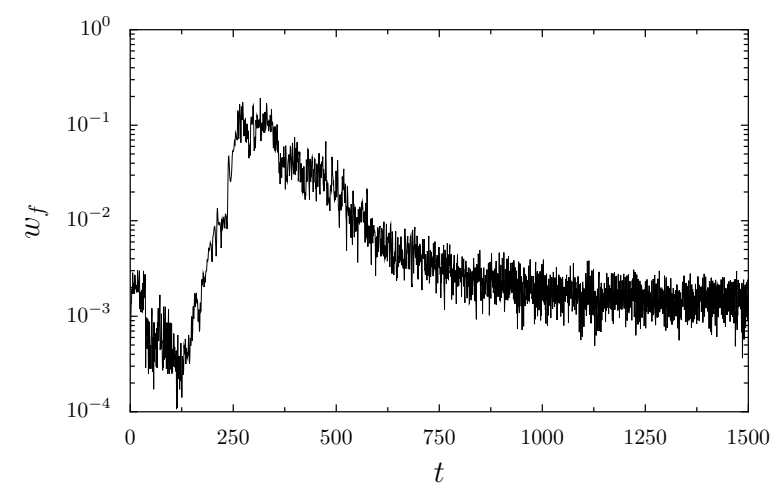

FIG. 1: Evolution of the field energy $w_{f}=\int\left(\partial_{x} \phi\right)^{2} d x$. Three phases can be distinguished: (a) an initial transient damping, (b) nonlinear instability and (c) saturation and decay towards a new equilibrium.

\section{A. Formation of solitary seed phase-space holes}

The dominant process in the earliest stage of the evolution is a kind of Landau damping where some of the noise is damped out due to phase mixing in the particle distribution functions. In the linear picture, Landau damping is standard textbook knowledge (see e.g. Ref. [18]), but in our case one has to admit that the fully nonlinear coupling between modes leads to a slightly different scenario in which incoherent noise is transformed into the coherent excitation of one or several small phase-space holes [19-21].

In all our simulations, we observe that coherent structures are created close to $v=0$ in the center of mass frame, which is the phase space region where particles of both species interact most strongly. Due to the symmetry of the system, holes in both species are equally probable. Therefore, without loss of generality, we focus on holes in the $f_{-}$distribution function.

To understand the existence and character of these solitary holes, we may adopt a quasistationary description of phase-space structures. Using the distribution functions and notation of Ref. [21] we can obtain equilibrium solutions of the VP system by requiring that the defining 
parameters satisfy the nonlinear dispersion relation (NDR)

$$
-Z_{r}^{\prime}\left(\frac{v_{D} / 2}{\sqrt{2}}\right)=\frac{16}{15}\left[b\left(\beta, v_{D} / 2\right)+\frac{3}{2} b\left(\alpha, v_{D} / 2\right)\right] \sqrt{\Psi}
$$

where we assumed that the structure is resting at the center of mass frame $\left(v_{0}=0\right)$ and that it is solitary $\left(k_{0}=0\right)$. The function $b(\beta, u)$, representing the trapping effect, is defined by

$$
b(\beta, u)=\frac{1}{\sqrt{\pi}}\left(1-\beta-u^{2}\right) \exp \left(-\frac{u^{2}}{2}\right)
$$

and $Z_{r}(x)$ stands for the real part of the plasma dispersion function. Note that (3) differs from the expression given in Ref. [6] because now we included a drift velocity $v_{D}$ and removed the additional assumption of complete symmetry between the species (i.e. we have $\alpha \neq \beta$ ).

If the seed hole is located in the $f_{-}$distribution function, it is a natural assumption that trapping in the positive species plays a minor role and therefore $b\left(\alpha, v_{D}\right) \approx 0$. Furthermore, if we take into account that for $v_{D}=2,-Z_{r}^{\prime}\left(\frac{v_{D} / 2}{\sqrt{2}}\right)>0$, then it must hold $b\left(\beta, v_{D} / 2\right)>0$. But from (4), this implies $\beta<0$, which means that only holes are allowed and no humps can appear. This is actually what we observed in all our numerical simulations.

\section{B. Acceleration and growth of holes}

A detailed look at the phase space densities in Fig. 2 of both species allows us to identify the nonlinear instability observed in Fig. 1 as the effect of the growth and multiplication of the phase space holes whose formation we described in the past section. This phenomenon was already observed by Berman et. al in their Particle-In-Cell (PIC) simulations [13]. Our Vlasov code and the use of equal masses of the two species allows us however to better understand the dynamics involved in the process and to improve the model proposed in Ref. [14].

Once a hole is created, it starts to accelerate with an almost constant acceleration. The mechanism involved in such a behavior consists in the formation of a hump in the distribution function of the opposite species $\left(f_{+}\right)$located slightly in front of the hole (see Fig. 2). The particles in that hump are bouncing in front of the hole as the acceleration of the latter always allows it to reach any preceding particle which is then in turn accelerated, thereby allowing the positive species to gain momentum from the negative one. Thus, a self-accelerating mechanism for phase-space holes is created. This mechanism can also be understood by transforming to a non-inertial frame comoving with the accelerated structure. In that frame we have to add a constant pseudo-force to the Vlasov equation and the trajectories of the particles in phase space look like those illustrated in 


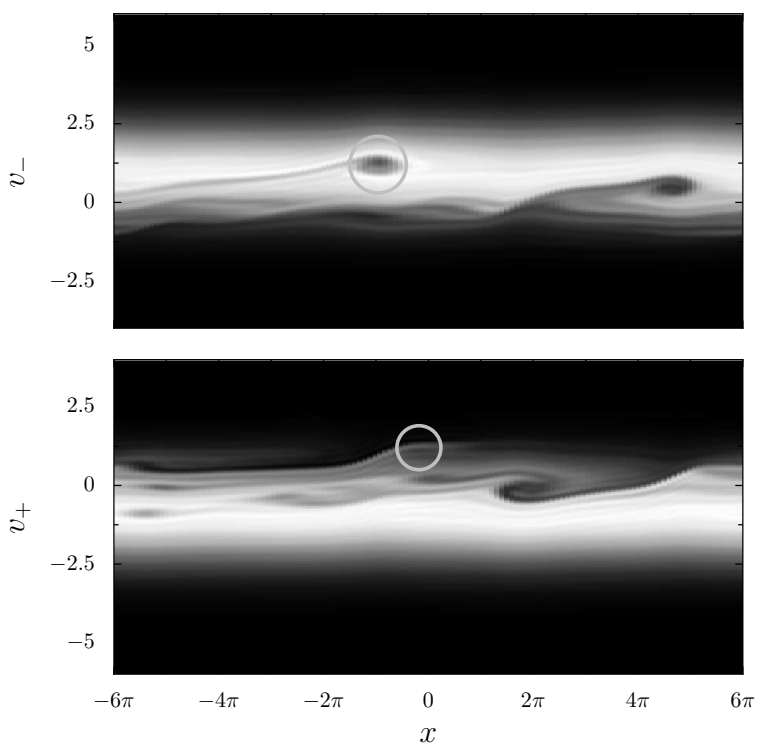

FIG. 2: Phase space distribution functions at $t=187.5$. The circles indicate the location of a hole in the $f_{-}$distribution function and the corresponding leading hump in the $f_{+}$function. In time, the hole tends to keep its shape while accelerating with a roughly constant acceleration.

Fig. 3. Here, we can see that in the new frame some positive particles are indeed trapped in front of the hole, which explains the leading hump that, in turn, is responsible for creating a positive net charge in front of the hole that attracts and accelerates the negative particles. In Fig. 4, the ion densities and the corresponding electrostatic potential that this configuration in phase space creates are shown. We can view the hole in the $f_{-}$distribution as a quasiparticle of positive charge and negative mass shielded by free negative particles $[14,20]$. Thus, due to the asymmetry of the potential seen in Fig. 4, the hole is sitting in a negative electric field and it is therefore accelerated to the right. As the free positive particles play no essential role, this mechanism can accelerate positive particles to velocities larger than their thermal range; this explains also our observation that the acceleration is roughly constant.

On the other hand, as the phase space densities inside the trapping areas remain approximately constant, the amplitude of the electrostatic potential grows as the hole penetrates into more populated regions in the phase space of negative particles reaching a maximum around $v_{0}=v_{D} / 2$. Later it decays and the hole finally disappears as $v_{0}-v_{D} / 2 \gg 1$.

The fluctuations in the electrostatic potential, created by the holes contribute in turn to trigger new seed holes. Thus, the rate of creation of holes is roughly proportional to the number of holes already present in the plasma. This multiplication of structures provides an explanation for the exponential growth that we can observe in the phase (b) of Fig. 1. After some time, the number 

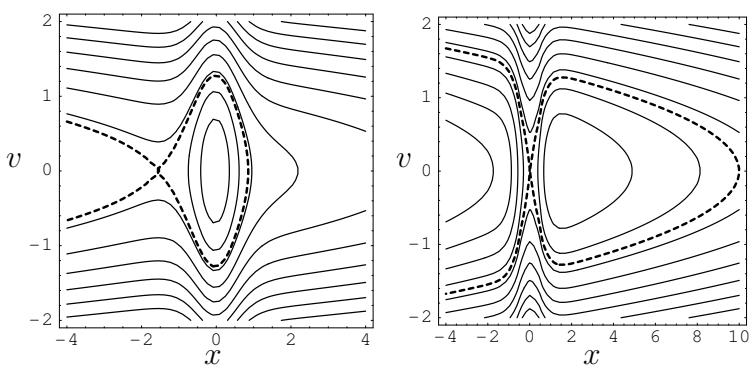

FIG. 3: Schematic phase space trajectories of negative (left) and positive (right) particles in a non-inertial frame, co-moving with a hole in the $f_{-}$distribution function (bell-shaped potential). The dashed line represents the separatrix between trapped and free particles in this frame.

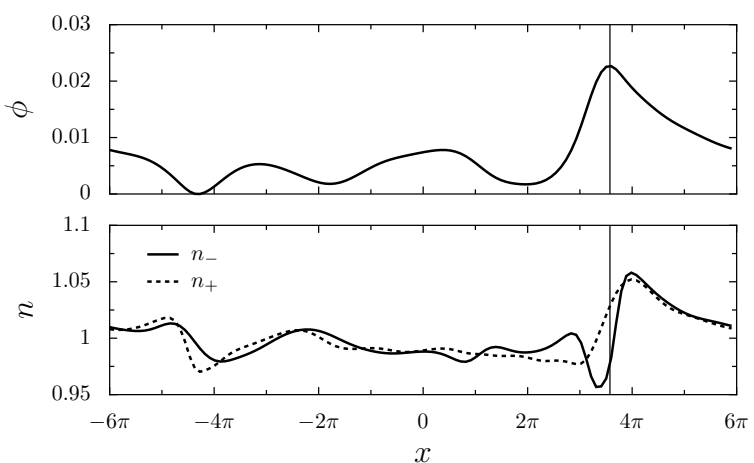

FIG. 4: Densities and electrostatic potential in an early stage of the evolution $(t=187.5)$. A hole in the negative particle species is seen to the right. Note the asymmetry and that the bottom of the negative density is shifted to the left of the maximum of the potential (marked by a vertical line), which induces an acceleration to the right. To the left in the figure, a smaller hole with negative acceleration can be seen in the positive species.

of holes is so large that interactions between them become the dominant process and the system reaches a chaotic, turbulent state in which the holes collide and merge.

\section{Saturation and stable equilibrium state}

After some stage of turbulence, the field energy of the system eventually saturates and the plasma slowly decays into a new equilibrium that seems to be stable. An examination of the phase space densities allows us to understand the involved kinetic processes. The relaxation set on by the present mechanism has the effect of widening the distribution functions and bringing them closer to one another or, to say it more formally, increasing the temperatures mainly at the cost of reducing the drift $v_{D}$ (see Fig. 5). This response can be viewed as the result of an effective 
collision between positive and negative plasma particles, mediated by the trapping structures as predicted by Korn and Schamel [10]. From a macroscopic viewpoint, it is understandable as the result of anomalous diffusion caused by the scattering of particles by trapping vortices [9, 10, 22].

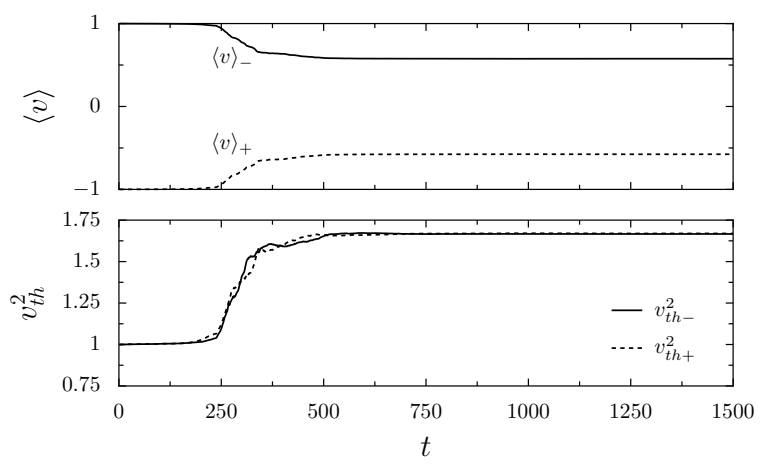

FIG. 5: Evolution of the mean velocity $\langle v\rangle$ (above) and the thermal speed squared $v_{t h}^{2}=\left\langle(v-\langle v\rangle)^{2}\right\rangle$ (below) of each species.

Note that friction does not conserve entropy, defined as $S=-\int d v f \log f$, and in principle it cannot be described by a Vlasov equation. We must remember, however, that neglecting some Fourier modes and Hermite polynomials, as we did in the decomposition (2), acts like a small dissipation and turns the Vlasov equation into a non entropy-conserving one. If we had been able to solve the complete Vlasov equation, the final distribution functions would have had oscillations of very high frequency in the velocity (filamentation), which in any realistic physical model should be averaged out.

The modifications of the distribution functions act against the formation and acceleration of holes, which therefore become less frequent as time advances. At some point, an equilibrium is reached which in most of our simulations consists in one of the species having a flat distribution function close to $v_{0}$, while the other one presents one or a few holes with constant speeds. It should be expected however that when more than one hole is present the coalescence instability would have acted, turning them into a single one after a long enough time. We note that this coalescence strongly depends on the length of the system in the sense that for a large $L$, a very long simulated time is needed to obtain a single hole.

Two sample scenarios are shown in Figs. 7 and 8. In Fig. 7, we show the phase space densities for an advanced state of the same run we use for the rest of the figures. Here, we see that three holes exist in the distribution function of the positive ions. As they have similar speeds, they spend most of the time very separated from each other, and therefore it will take a very long time until a single hole appears. On the other hand, in Fig. 8 we show the phase space densities at an 
advanced time of a different run with a smaller box length. Here, a single hole appears in the $f_{+}$ distribution function, while in the $f_{-}$distribution, a plateau is seen with an embedded hole that, due to the repulsion with the hole in the $f_{+}$species, slowly oscillates back and forth.

In Fig. 6, we show the initial and final distribution functions, corresponding to Fig. 7, integrated in space. A depression is clearly seen in the distribution function of positive ions and a corresponding flat region in $f_{-}$. The flattening prevents that the hole is accelerated into higher speeds.

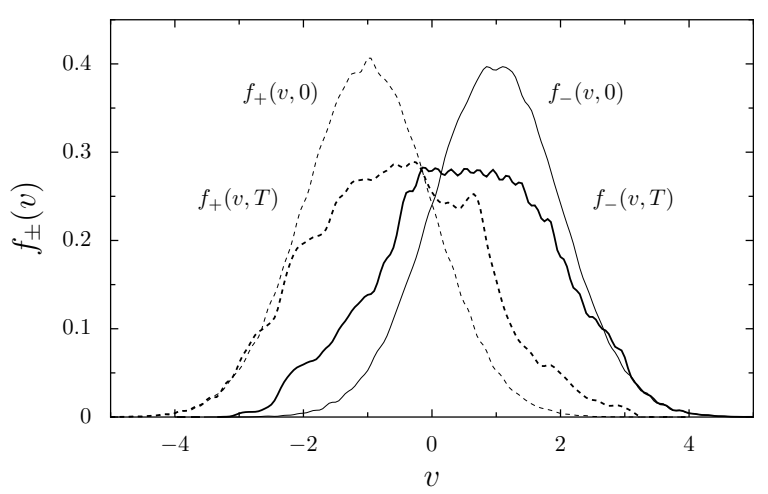

FIG. 6: Velocity distribution functions at $t=0$ and at $t=T=1500$.

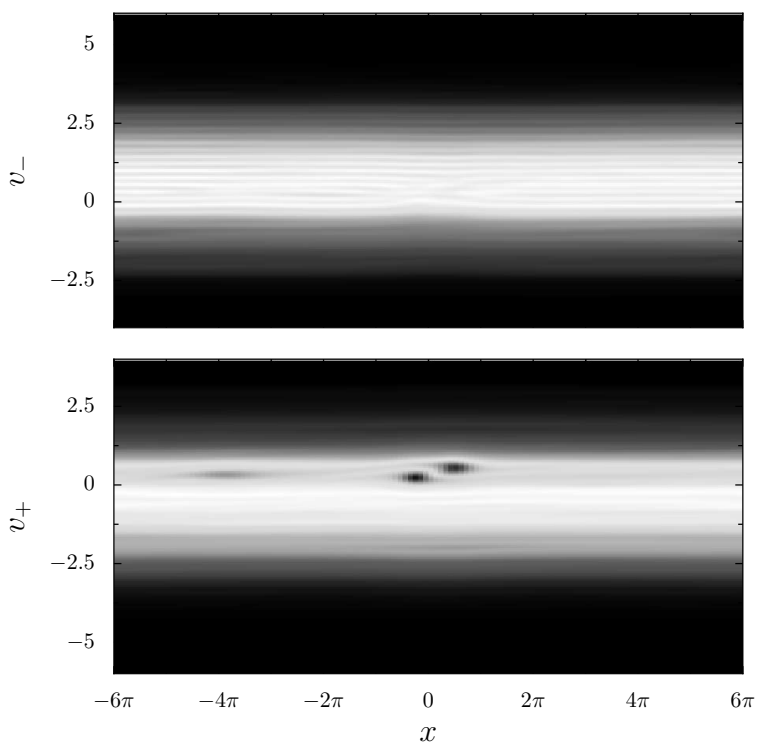

FIG. 7: Phase space densities at $t=1350$. The system at this point is almost stationary, where $f_{-}$has a flat plateau and $f_{+}$has three holes which are propagating with very close speeds. For a much larger time, it is expected that the three holes coalesce and form a solitary one. 


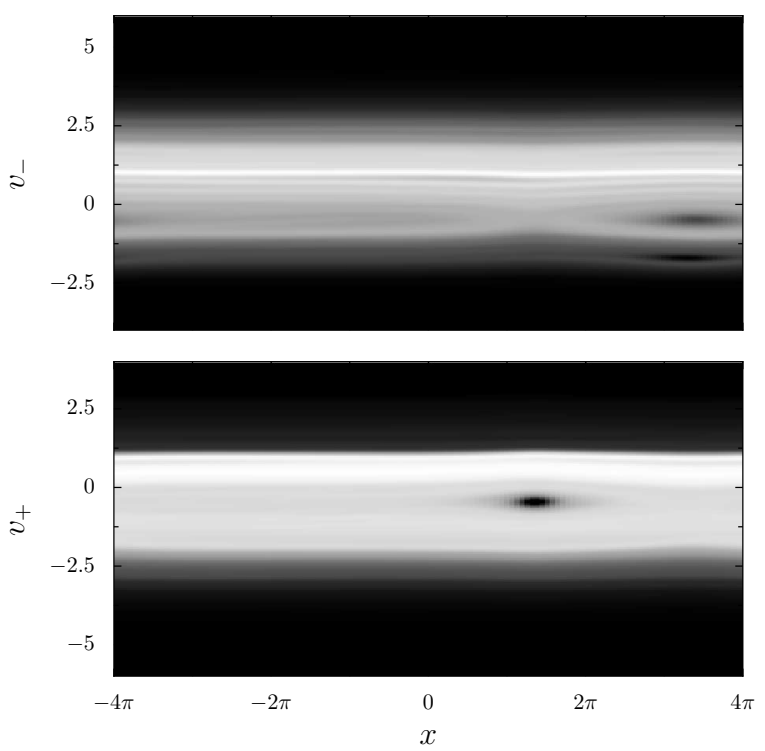

FIG. 8: Phase space densities at $t=1350$ for a run with a smaller system length. In this case a single hole is present in $f_{+}$, while a plateau with an embedded hole appears in $f_{-}$.

\section{SUMMARY AND CONCLUSIONS}

In this paper, the nonlinear stability of a two-species current-carrying pair plasma described by the Vlasov-Poisson system has been investigated in the linearly stable regime. By numerical simulations, we have shown that the system can be destabilized by incoherent, topologically deforming perturbations. We have explained qualitatively the process involved in this nonlinear destabilization, which consists of the spontaneous creation of holes in the particle distribution function of one species and associated humps in the opposite species, and their self-acceleration. An analytical model for such mechanism would be highly desirable, as it may allow to estimate the threshold drift of the nonlinear stability. Whether zero or negative energy arguments [23] will play a role in such a model is another question that should be addressed in forthcoming investigations.

We have also shown that the nonlinear destabilization and subsequent turbulent stage drives the system towards a new stable equilibrium in which quasistationary holes are present. In a forthcoming publication, use will be made of the distribution functions of Ref. [21] to analyze these final steady states.

We note that some points remain to be clarified. Among them, a description of the processes involved in the saturation of the nonlinear stability, probably based in a minimization principle of some sort of free energy, seems to be the most challenging one. An analytical approach to the study of the stability of seed holes, on the other hand, would allow to estimate the nonlinear stability 
threshold, which is much lower than the linear one. This would surely be a remarkable step in plasma physics.

Finally, we would like to suggest the possibility that an experimental setup like the one presented in Ref. [5] be slightly modified to include a drift between both species. These highly valuable experiments would provide a new insight into nonlinear current-driven instability and, in general, contribute to the understanding of stability and transport issues in plasmas with electrons and anti-electrons or positive and negative ion components.

As a conclusion, we would like to emphasize the fact that our simulations prove once more that the smallness of a perturbation is a necessary but not a sufficient condition to justify the linearization of the Vlasov-Poisson system. Instead, nonlinear considerations have to be taken into account from the very beginning and this paper shows another mechanism by which the nonlinear trapping phenomena are the key to the global dynamics of a plasma.

\section{Acknowledgments}

This work was supported by the European Commission (Brussels) through contract No. HPRNCT-2001-00314 for carrying out the task of the RTN Network "Turbulent Boundary Layers in Geospace Plasmas", as well as by the Deutsche Forschungsgemeinschaft through the Sonderforschungsbereich 591 .

[1] J. Vranjes, and S. Poedts, Plasma Sources Sci. Technol. 14, 485 (2005).

[2] C. M. Surko, M. Leventhal, and A. Passner, Phys. Rev. Lett. 62, 901 (1989).

[3] H. Boehmer, M. Adams, and N. Rynn, Phys. Plasmas 2, 4369 (1995).

[4] E. P. Liang, S. C. Wilks, and M. Tabak, Phys. Rev. Lett. 81, 4887 (1998).

[5] W. Oohara and R. Hatakeyama, Phys. Rev. Lett. 91, 205005 (2003).

[6] H. Schamel and A. Luque, New J. Phys. 7, 69 (2005).

[7] H. Schamel and A. Luque, Nonlinear growth of trapped particle modes in linearly stable current-carrying plasmas, Space Sci. Rev., (submitted).

[8] A. Luque and H. Schamel, Phys. Rep., 415, 261 (2005).

[9] J. Korn and H. Schamel, J. Plasma Phys. 56, 307 (1996).

[10] J. Korn and H. Schamel, J. Plasma Phys. 56, 339 (1996).

[11] B. D. Fried and R. W. Gould, Phys. Fluids 4, 139 (1961).

[12] N. J. Balmforth and R. R. Kerswell, J. Plasma Phys. 68, 87 (2002). 
[13] R. H. Berman, D. J. Tetreault, T. H. Dupree, and T. Boutros-Ghali, Phys. Rev. Lett. 48, 1249 (1982).

[14] T. H. Dupree, Phys. Fluids 25, 277 (1982).

[15] M. Shoucri and G. Knorr, J. Chem. Phys. 14, 84 (1974).

[16] T. O'Neil, Phys. Fluids 8, 2255 (1965).

[17] G. Manfredi, Phys. Rev. Lett. 79, 2815 (1997).

[18] N. A. Krall and A. W. Trivelpiece, Principles of Plasma Physics (McGraw-Hill, New York, 1973).

[19] H. Schamel, Physica Scripta 20, 306 (1979).

[20] H. Schamel, Physics Reports 140, 161 (1986).

[21] H. Schamel, Phys. Plasmas 7, 4831 (2000).

[22] H. Schamel and J. Korn, Physica Scripta T63, 63 (1996).

[23] J.-M. Grießmeier, A. Luque, and H. Schamel, Phys. Plasmas 9, 3816 (2002). 